Pacific Journal of Mathematics

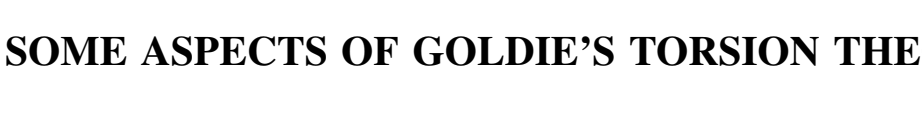




\title{
SOME ASPECTS OF GOLDIE'S TORSION THEORY
}

\author{
MARK L. TePly
}

\begin{abstract}
Goldie's torsion class $\mathscr{C}$ is a class of left $R$-modules closed under taking submodules, factor modules, extensions, arbitrary direct sums, and injective envelopes. The corresponding Goldie torsionfree class $\mathscr{F}$ is precisely the class of left $R$-modules possessing zero singular submodule. It is shown that $\mathscr{G}$ is closed under taking direct products if and only if nonzero left ideals in $\mathscr{F}$ have nonzero socles. Another theorem gives four conditions equivalent to the following: Any direct sum of torsionfree injective modules is injective. One of these four conditions is that the ring $R$ is an essential extension of a finite direct sum $\mathscr{G}(R) \oplus L_{1} \oplus L_{2} \oplus \cdots \oplus L_{n}$, where each $L_{i}$ is a uniform left ideal of $R$. It is natural to ask when $R$ actually equals this direct sum. A sufficient condition for this to happen is given. Rings in which every torsionfree principal left ideal is projective are studied. Particular attention is paid to those rings whose Goldie torsion filters possess a cofinal subset of finitely generated left ideals.
\end{abstract}

In this paper all rings $R$ are associative with unit, and all modules are unitary left $R$-modules.

Before proceeding, we review some results from [2]. The Goldie torsion class is the smallest class of modules which is closed under taking factor modules, extensions, and arbitrary direct sums, and which contains all factor modules $B / A$, where $A$ is an essential submodule of $B$. Then the class $\mathscr{F}=\left\{F \in \in_{R} \mathscr{C} \mid \operatorname{Hom}_{R}(G, F)=0\right.$ for all $G \in \mathscr{C}\}$ is a torsionfree class in the sense of $[5]$. Moreover, ( $\mathscr{G}$, $\mathscr{F}$ ) is a torsion theory in the sense of [5], $\mathscr{G} \cap \mathscr{F}=0$, and every $M \in_{R} \mathscr{C l}$ has a (necessarily unique) maximal torsion submodule $\mathscr{G}(M)$ such that $M \cdot / \mathscr{G}(M) \in \mathscr{F}$. $\mathscr{F}$ is precisely the class of $R$-modules which have zero singular submodule; moreover, $\mathscr{F}$ is closed under taking: submodules, direct products, extensions, and injective envelopes. In particular, a left ideal $I$ of $R$ is in $\mathscr{F}$ if and only if $I$ has zero singular submodule when considered as a left $R$-module. In case $R$ is an integral domain, then $\mathscr{G}$ coincides with class of modules which are torsion in the usual sense.

Associated with $\mathscr{G}$ there is a filter of left ideals $F(\mathscr{G})=$ $\{L \mid R / L \in \mathscr{G}\}$. In [1] J. S. Alin shows $L \in F(\mathscr{C})$ if and only if there exists $L^{\prime}$ essential in $R$ such that $L \cong L^{\prime}$ and $(L: x)$ is essential in $R$ for all $x \in L^{\prime}$. In particular, every essential left ideal of $R$ is in $F(\mathscr{G})$. As in [14], $F(\mathscr{G})$ is said to have a cofinal subset of finitely generated left ideals if, given $L \in F(\mathscr{C})$, there exists $I \subseteq L$ such that $I$ is finitely 
generated and $I \in F(\mathscr{C})$.

When we say a module $G$ is "torsion," then we mean $G \in \mathscr{G}$; when we say a module $F$ is torsionfree, we mean $F \in \mathscr{F}$.

An $R$-module $M$ will be called uniform if, for any pair $L, N$ of nonzero submodules of $M, L \cap N \neq 0$. A left ideal of $R$ is called uniform if it is uniform as a left $R$-module.

1. Products of Goldie torsion modules. In [9] J. P. Jans investigated classes of modules which are closed under submodules, homomorphic images, extensions, and direct products. Such classes are called torsion-torsionfree classes (TTF classes). It is clear that the Goldie torsion class will be a TTF class if and only if $\mathscr{G}$ is closed under direct products. R. S. Pierce has pointed out ([9], Th. 2.1) that $\mathscr{G}$ is closed under products if and only if $I=\bigcap_{L \in F(\mathscr{G})} L \in F(\mathscr{G})$. In that case $I$ is a two-sided idempotent ideal of $R$. In studying the simple torsion class $\mathscr{S}$ of S. E. Dickson [5], J. S. Alin has shown [1] that if $\mathscr{S}$ is closed under direct products, then $\mathscr{G} \cap \mathscr{S}$ is closed under direct products. If nonzero modules have nonzero socles, then $\mathscr{S}=$ ${ }_{R} \mathscr{C l}$, and hence Alin's results shows $\mathscr{G}$ is closed under direct products. This motivates the main result of this section (Th. 1.3).

Since the ideal $I=\bigcap_{L \in F(\mathscr{O})} L$ plays a key role in examining Goldie torsion classes closed under direct products, we begin this section by examining $I$.

\section{Proposition 1.1. Let $I=\bigcap_{L \in F(\mathscr{\vartheta})} L$. Then $I \in \mathscr{F}$.}

Proof. By Zorn's lemma, there is a left ideal $J$ maximal with respect to $J \cap \mathscr{G}(I)=0$. Then $J+\mathscr{G}(I)$ is essential in $R$. Since $\mathscr{G}$ is closed under extensions, then the exact sequence

$$
0 \rightarrow \frac{J+\mathscr{C}(I)}{J} \rightarrow \frac{R}{J} \rightarrow \frac{R}{J+\mathscr{C}(I)} \rightarrow 0
$$

yields $R / J \in \mathscr{G}$, i.e., $J \in F(\mathscr{G})$. Thus by the definition of $I, J \supseteqq I \supseteqq$ $\mathscr{G}(I)$, and hence $\mathscr{G}(I)=0$.

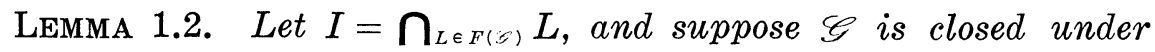
direct products. Then:

(1) If $J$ is a left ideal of $R$ and $J \subseteq I$, then $I=J \oplus K$ for some left ideal $K$.

(2) If $I \neq 0$, then $I=\bigoplus \sum_{\alpha \in \mathfrak{A}} S_{\alpha}$ where $S_{\alpha}$ is simple and $\mathfrak{A}$ is an index set.

Proof. Let $J \subseteq I$ be a left ideal of $R$. There exists a left ideal 
$K$ of $R$ maximal with respect to the properties $K \cap J=0$ and $K \subseteq I$. Then $K \oplus J$ is essential in $I$ and hence $I / K \oplus J \in \mathscr{C}$. By [9] Th. 2.1, $I \in F(\mathscr{O})$; so the exact sequence

$$
0 \rightarrow I / J \oplus K \rightarrow R / J \oplus K \rightarrow R / I \rightarrow 0
$$

yields $R / J \oplus K \in \mathscr{G}$, i.e., $J \oplus K \in F(\mathscr{G})$. It follows from the definition of $I$ that $J \oplus K \supseteqq I$, and hence $I=J \oplus K$.

(2) follows from (1) and [4], Theorem 15.3.

THEOREM 1.3. The following are equivalent:

(1) $\mathscr{G}$ is closed under direct products.

(2) Nonzero modules in $\mathscr{F}$ have nonzero socles.

(3) Nonzero left ideals in $\mathscr{F}$ have nonzero socles.

Proof. $\quad(1) \Rightarrow(2):$ Suppose $F \in \mathscr{F}$ and $0 \neq x \in F$. By [9] Theorem 2.1 and (1), $I \in F(\mathscr{C})$. Claim $I x \neq 0$; for otherwise $I \in F(\mathscr{C})$ implies $R x \in \mathscr{G}$, and $R x \subseteq F$ implies $R x \in \mathscr{G}$. Thus $R x \in \mathscr{C} \cap \mathscr{F}=0$, a contradiction. By Lemma 1.2, it then follows that $S_{\alpha} x \neq 0$ for some simple left ideal $S_{\alpha}$. Then $S_{\alpha} x$ is a nonzero homomorphic image of $S_{\alpha}$, and hence is a simple submodule of $F$. Thus $F$ has nonzero socle.

$(2) \Rightarrow(3)$ : Trivial.

( 3$) \Rightarrow(1)$ : If $R \in \mathscr{G}$, then $\mathscr{G}={ }_{R} / \mathscr{l}$, and so the result is trivially true. Suppose $R \notin \mathscr{G}$. Since $\mathscr{G}$ is closed under essential extensions, there exists $K \subseteq R$ such that $K \in \mathscr{F}$. By (3) there exists $S \subseteq K$ with $S$ simple.

Claim that $L \in F(\mathscr{C})$ implies $S \subseteq L$. For if $L \cap S=0$, then $S \cong S+L / L \cong R / L \in \mathscr{G}$; and hence $S \in \mathscr{G}$, contradicting $S \in \mathscr{F}$. But then $L \cap S \neq 0$ implies $S \subseteq L$. Since $L \in F(\mathscr{C})$ was arbitrarily chosen, then $S \subseteq \bigcap_{L \in F(\Im)} L=I$. Therefore $K \cap I \neq 0$.

Let $J$ be a left ideal of $R$ maximal with respect to $J \cap I=0$. By the previous paragraph $J$ contains no left ideals in $\mathscr{F}$; hence $\mathscr{G}(J)$ is essential in $J$. Therefore $J \in \mathscr{G}$. So the exact sequence

$$
0 \rightarrow \frac{J+I}{I} \rightarrow \frac{R}{I} \rightarrow \frac{R}{I+J} \rightarrow 0
$$

yields $R / I \in \mathscr{G}$, i.e., $I \in F(\mathscr{G})$. Hence $\mathscr{C}$ is closed under direct products by [9], Theorem 2.1.

In [1] J. S. Alin points out that every simple module in $\mathscr{F}$ is projective. Hence if $R$ has no projective simples and $\mathscr{G}$ is closed under directs products, Proposition 1.1 and Lemma 1.2 imply $I=0$. Thus we obtain: 
Proposition 1.4. Suppose $R$ has no projective simples. Then $\mathscr{G}$ is closed under direct products if and only if $R \in \mathscr{G}$.

It is known [2] that the following are equivalent: (1) $R=\mathscr{G}(R)+S$ (ring direct sum) where $S$ is semisimple with minimum condition; (2) $\mathscr{F}$ is closed under homomorphic images; and (3) the Goldie global dimension of $R$ (see [2]) is zero. Thus it is of interest to examine these rings in relation to the condition: $\mathscr{G}$ is closed under direct products.

Proposition 1.5. $R=\mathscr{G}(R)+S$ (ring direct sum) where $S$ is semisimple with minimum condition if and only if the following conditions are satisfied:

(1) $\mathscr{C}$ is closed under products.

(2) $I=\bigcap_{L \in F(S)} L$ is finitely generated as a left ideal.

(3) There are no nonzero nilpotent left ideals in $\mathscr{F}$.

Proof. $\quad(\Longleftarrow)$ : If $R$ has no projective simple modules, then we are done by Proposition 1.4. Let $R=S_{1} \oplus M_{1}$ with $S_{1}$ simple. If $I=\bigcap_{L \in F(\xi)} L$, then $S_{1} \subseteq I$ follows from (1). Hence $I=S_{1} \oplus\left(M_{1} \cap I\right)$. If $M_{1} \cap I$ contains a simple summand $S_{2}$ of $R$, then it follows that $R=S_{1} \oplus S_{2} \oplus\left(M_{1} \cap M_{2}\right)$ where $R=S_{2} \oplus M_{2}$. Proceeding by induction $R=S_{1} \oplus S_{2} \oplus \cdots \oplus S_{n} \oplus\left(\bigcap_{i=1}^{n} M_{i}\right)$. Now this induction process must stop after a finite number of steps by (2), say

$$
R=S_{1} \oplus \cdots \oplus S_{N} \oplus\left(\bigcap_{i=1}^{N} M_{i}\right)
$$

where $I \cap\left(\bigcap_{i=1}^{N} M_{i}\right)$ contains no simple summands of $R$. Set $\bigcap_{i=1}^{N} M_{i}=G$. We claim that $I \cap G=0$. For otherwise (1) and Theorem 1.3 imply there exists a simple module $S \subseteq I \cap G . \quad S^{2} \neq 0$ by (3) and Proposition 1.1. Let $x, y \in S$ such that $x y \neq 0$. Then $y$ generates $S$, and $(0: y) \cap S=0$ since $S$ is simple. But $(0: y)$ is maximal, and hence $(0: y) \oplus S=R$, contradicting $I \cap G$ contains no simple summands of $R$. Hence $I \cap G=0$ as claimed, and so $I=S_{1} \oplus \cdots \oplus S_{N}$.

Now observe that $G=\mathscr{C}(R)$ as follows: Clearly $\mathscr{G}(R)$ cannot properly contain $G$. On the other hand, $G=I+G / I \subseteq R / I \in \mathscr{C}$ by (1), and hence $G \in \mathscr{C}$. Therefore, $G=\mathscr{C}(R)$.

Since $R=G \oplus I$ and since $G$ and $I$ are two-sided ideals, then $R=G+I$ (ring direct sum.)

$(\Rightarrow)$ : If $R=\mathscr{C}(R)+S$, where $S$ is semisimple with minimum conditions, then [2], Theorem 3.1. and the remark following [2], Corollary 3.4, show $\mathscr{C}$ is closed under direct products.

2. Direct sums of torsionfree injectives. The main theorem 
of this section points out the relationship between cofinal subsets of finitely generated left ideals in $F(\mathscr{G})$ and properties of sums of torsionfree injective modules. This relationship is also studied in [13]. An example in [13] shows that, for more general torsion theories [5] than the Goldie theory, the analogue of Theorem $2.1(1) \Rightarrow(5)$ is not always true. In [7], Theorem 5.6, a ring with $\mathscr{G}(R)=0$ and the ascending chain condition is represented as an essential extension of a finite direct sum. Condition (3) of Theorem 2.1 represents more general rings in this way.

THEOREM 2.1. The following are equivalent:

(1) $F(\mathscr{G})$ has a cofinal subset of finitely generated left ideals.

(2) $R$ contains no infinite direct sum of torsionfree left ideals.

(3) $\quad R$ is an essential extension of $\mathscr{G}(R) \oplus L_{1} \oplus L_{2} \oplus \cdots \oplus L_{n}$, where each $L_{i}$ is a uniform left ideal.

(4) $R / \mathscr{G}(R) \cong K$ where $K$ is an essential extension of a direct sum of finitely many uniform torsionfree left ideals.

(5) Any direct sum of torsionfree injective modules is injective.

REMARK. [13], Theorem 1.2, gives eight additional conditions which are equivalent to (5) (above) for more general hereditary torsion theories in the sense of [1], [9], and [13].

Proof. $\quad(1) \Longrightarrow(2)$ : Let $\oplus \sum_{\alpha \in w} L_{\alpha}$ be a direct sum of nonzero torsionfree left ideals of $R$. Then there exists a left ideal $L$ of $R$ such that $L \cap\left(\sum_{\alpha \in w} L_{\alpha}\right)=0$ and $V=L \oplus\left(\sum_{\alpha \in w} L_{\alpha}\right)$ is essential in $R$. Then $V \in F(\mathscr{G})$, so (1) implies that there exists $N \cong V$ with $N \in F(\mathscr{G})$ and $N$ finitely generated. Now each generator $N$ has a nonzero representation in only finitely many coordinants of $V=L \oplus\left(\oplus \sum_{\alpha \in w} L_{\alpha}\right)$. Since $N$ is finitely generated, it follows that there exists $u \leqq w$ such that $|u|<\boldsymbol{\aleph}_{0}$ and $N \cong L \oplus \sum_{\alpha \in u} L_{\alpha}$. Suppose $|u|<|w|$. Then

$$
\bigoplus_{\alpha \in w-u} L_{\alpha} \cong \frac{V}{L \oplus\left(\oplus \sum_{\alpha \in u} L_{\alpha}\right)} \cong \frac{R}{L \oplus\left(\bigoplus \sum_{\alpha \in u} L_{\alpha}\right)} \in \mathscr{G}
$$

since $N \subseteq L \oplus\left(\oplus \sum_{\alpha \in u} L_{\alpha}\right)$ implies $L \oplus\left(\oplus \sum_{\alpha \in u} L_{\alpha}\right) \in F(\mathscr{G})$. Hence $\bigoplus \sum_{\alpha \in w-u} L_{\alpha} \in \mathscr{G} \cap \mathscr{F}=0$, contradicting $L_{\alpha} \neq 0$. Hence $|w|=$ $|u|<\boldsymbol{\aleph}_{0}$, establishing (2).

$(2) \Rightarrow(5)$ : Consider the diagram:

( c )

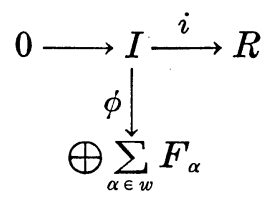


where $I$ is a left ideal of $R$ and $\left\{F_{\alpha}\right\}_{\alpha \in w}$ is a set of torsionfree injective $R$-modules. Note that $\phi(\mathscr{G}(I)) 0$. If $I$ is an essential extension of $\mathscr{G}(I)$, then $I=\mathscr{G}(I)$ since $\mathscr{G}$ is closed under essential extensions; but then the zero map from $R$ to $\oplus \sum_{\alpha \in w} F_{\alpha}$ makes (c) commute.

If $I$ is not an essential extension of $\mathscr{G}(I)$, then by (2) there exists a finite direct sum $\oplus \sum_{i=1}^{n} R x_{i}$ contained in $I$ maximal with respect to $\mathscr{G}(I) \cap\left(\oplus \sum_{i=1}^{n} R x_{i}\right)=0$. Then $U=\mathscr{G}(I) \oplus\left(\oplus \sum_{i=1}^{n} R x_{i}\right)$ has the properties: $I / U \in \mathscr{G}$ and $\phi(U)$ is contained in a direct sum of finitely many $F_{\alpha}^{\prime} \mathrm{s}(\alpha \in w)$. So by the injectivity of the $F_{\alpha}^{\prime} \mathrm{s}(\alpha \in w)$, there exists $f: R \rightarrow \bigoplus \sum_{\alpha \in w} F_{\alpha}$ such that $f|U=\phi| U$. Since $(f-\phi)(U)=0$, there is an induced map $g: I / U \rightarrow \bigoplus \sum_{\alpha \in w} F_{\alpha}$ via $g(x+U)=(f-\phi)(x)$. Since $I / U \in \mathscr{G}$ and $F_{\alpha} \in \mathscr{F} \forall \alpha$, then $g=0$, and hence $f \mid I=\phi$. Hence $f$ makes (c) commute, and thus $\oplus \sum_{\alpha \in w} F_{\alpha}$ is injective.

$(5) \Rightarrow(1):$ [13], Theorem 1.5.

$(2) \Rightarrow(3)$ : By (2) let $\oplus \sum_{i=1}^{n} L_{i}$ be a maximal direct sum of torsionfree left ideals of $R$. From (2) it follows that each torsionfree left ideal contains a uniform left ideal. So by breaking the $L_{i}$ apart into direct subsums, we may assume each $L_{i}$ is uniform. By the maximal property of $\oplus \sum_{i=1}^{n} L_{i}$, it follows that $\mathscr{G}(R) \oplus\left(\oplus \sum_{i=1}^{n} L_{i}\right)$ is essential in $R$.

( 3$) \Rightarrow(4)$ : If $M \nsubseteq \mathscr{G}(R)$, we claim $M \cap \sum_{i=1}^{n} L_{i} \neq 0$. For suppose $M \nsubseteq \mathscr{G}(R)$ and $M \cap\left(\sum_{i=1}^{n} L_{i}\right)=0$. Then let

$$
0 \neq x=g+r_{1}+r_{2}+\cdots+r_{n} \in\left(\mathscr{G}(R) \oplus\left(\sum_{i=1}^{n} L_{i}\right)\right) \cap M
$$

by (3). Then $g \neq 0$. If $(0: g) x \neq 0$, then $M \cap \sum_{i=1}^{n} L_{i} \neq 0$, a contradiction to our assumption. Hence $(0: g) x=0$, and so $x \in \mathscr{G}(M)$. Thus $M \cap\left(\sum_{i=1}^{n} L_{i} \oplus \mathscr{G}(R)\right) \in \mathscr{G}$. From (3), $M \cap\left(\sum_{i=1}^{n} L_{i} \oplus \mathscr{G}(R)\right)$ is essential in $M$, and hence $M \in \mathscr{G}$ by $\mathscr{G}$ closed under essential extensions. Thus $M \subseteq \mathscr{G}(R)$, which is a contradiction to our choice of $M$.

From the claim, it follows that $R / \mathscr{G}(R) \cong K$ is an essential extension of $\left(\oplus \sum_{i=1}^{n} L_{i}\right) \oplus \mathscr{G}(R) / \mathscr{G}(R) \cong \bigoplus \sum_{i=1}^{n} L_{i}$ with $L_{i}$ uniform.

$(4) \Rightarrow(1)$ : Assume $R \notin \mathscr{G}$. Let $K$ be an essential extension of $\oplus \sum_{i=1}^{n} L_{i}, L_{i}$ uniform left ideals; $K=R / \mathscr{G}(R)$ by (4). If $I \in F(\mathscr{G})$, then $I \cap L_{i} \neq 0$ since $L_{i} \in \mathscr{F}$. Choose $0 \neq x_{i} \in I \cap L_{i}$ for $i=1,2, \cdots, n$. Since $L_{i}$ is uniform, $R x_{i}$ is essential in $L_{i}$. Hence

$$
\frac{\bigoplus \sum_{i=1}^{n} L_{i}}{\bigoplus \sum_{i=1}^{n} R x_{i}} \cong \bigoplus \sum_{i=1}^{n} L_{i} / R x_{i} \in \mathscr{G} .
$$

So the exact sequence 


$$
0 \rightarrow \frac{\mathscr{C}(R) \oplus \sum_{i=1}^{n} L_{i}}{\bigoplus \sum_{i=1}^{n} R x_{i}} \rightarrow \frac{R}{\bigoplus \sum_{i=1}^{n} R x_{i}} \rightarrow \frac{R}{\mathscr{G}(R) \oplus \sum_{i=1}^{n} L_{i}} \rightarrow 0
$$

yields $R / \sum_{i=1}^{n} R x_{i} \in \mathscr{C}$, i.e., $\sum_{i=1}^{n} R x_{i} \in F(\mathscr{C})$.

The following result of F. L. Sandomierski ([12], Th. 2.5) is an immediate corollary of Theorem $2.1(2) \Rightarrow(5)$ :

COROLlary 2.2. If $R$ has no infinite direct sums of left ideals, then any direct sum of injective $R$-modules with zero singular submodule is injective.

ExAMPLE. To see that $R$ can have an infinite direct sum of left ideals without having an infinite direct sum of torsionfree left ideals, consider the ring $R=\left[\prod_{\alpha \in w} P^{(\alpha)}\right]+N$ (ring direct sum) where $|w| \geqq$ $\aleph_{0}, P^{(\alpha)}=Z /\left(p^{2}\right)(Z=$ integers, $p=$ prime $)$, and $N$ is a commutative Noetherian ring with zero singular ideal. Then $\mathscr{G}(R)=\prod_{\alpha \in w} P^{(\alpha)}$, and $R$ clearly has infinite direct sums of left ideals. But since $R$ is commutative and $N \in \mathscr{F}$ is Noetherian, $R$ contains no infinite direct sums of torsionfree left ideals. Thus Theorem 2.1 gives a proper generalization of Sandomierki's result.

If $R$ has zero singular ideal, then $F(\mathscr{G})=\{I \mid I$ is an essential left ideal of $R$. Hence the following result of C. Walker and E. A. Walker ([14], Th. $4.20(\mathrm{~b}) \Leftrightarrow(\mathrm{c}))$ is an immediate corollary of Theorem $2.1(1) \Leftrightarrow(2)$.

CoRollary 2.3. Let $R$ have zero singular ideal, and let $F(\mathscr{G})$ be the filter of all essential left ideals. Then the following are equivalent:

(1) $F(\mathscr{G})$ has a cofinal subset of finitely generated left ideals.

(2) $R$ has no infinite direct sums of left ideals.

3. $(\mathscr{F} P P)$ rings. From Theorem 2.1 we see that the condition, $F(\mathscr{G})$ has a cofinal subset of finitely generated left ideals, is equivalent to $R$ being an essential extension of the finite direct sum

$$
\mathscr{G}(R) \oplus L_{1} \oplus L_{2} \oplus \cdots \oplus L_{n},
$$

where each $L_{i}$ is a uniform left ideal of $R$. It is a natural question to ask when $R$ is actually equal to this direct sum. The condition that torsionfree principal left ideals are projective plays an interesting role as a sufficient condition for equality. The main purpose of this section is to examine this role and hence to obtain generalizations of some results of L. Levy [11] and A. Hattori [8]. 
A ring $R$ is called $(\mathscr{F} P P$ ) if every torsionfree principal left ideal is projective. This is a generalization of Hattori's concept of $(P P)$ ring [8]. There a ring is called $(P P)$ if every principal left ideal is projective. It is easily seen that a ring $R$ is $(P P)$ if and only if $R$ is $(\mathscr{F} P P)$ and $\mathscr{G}(R)=0$.

THEOREM 3.2. Let $R$ be an $(\mathscr{F} P P)$ ring. Then the following are equivalent:

(1) $F(\mathscr{G})$ has a cofinal subset of finitely generated left ideals.

(2) $R=R e_{1} \oplus R e_{2} \oplus \cdots \oplus R e_{n} \oplus A$, where each $R e_{i}$ is a uniform left ideal and where $A$ is an essential extension of

$$
\mathscr{G}(R) \oplus N_{1} \oplus N_{2} \oplus \cdots \oplus N_{m} \text {. }
$$

$N_{i}^{2}=0$ for $i=1,2, \cdots, m$, and each $N_{i}$ is $R$-isomorphic with some $R e_{j(i)}$ where $j(i) \in\{1,2, \cdots, n\}$. Moreover, $1=e_{1}+e_{2}+\cdots+e_{n}+a$, $a \in A$, and $a N_{i}=0$ for $i=1,2, \cdots, m$.

Proof. $\quad(1) \Rightarrow(2)$ : If $R=\mathscr{G}(R)$, then there is nothing to prove. If $R \neq \mathscr{G}(R)$, then there exists a left ideal $I$ such that $I \cap \mathscr{G}(R)=0$ since $\mathscr{G}$ is closed under essential extensions. By (1) and Theorem 2.1 (2) we may assume $I=R x$ is a uniform left ideal in $\mathscr{F}$. Now the exact sequence

$$
0 \rightarrow(0: x) \rightarrow R \rightarrow R x \rightarrow 0
$$

must split since $R$ is $(\mathscr{F} P P)$. Hence $R=D_{1} \oplus A_{1}$ where $D_{1} \cong R x$. Write $1=e_{1}+a_{1}$ where $e_{1} \in D, a_{1} \in A_{1}$. Then $D_{1}=R e_{1}$. Since $\mathscr{G}$ is closed under homomorphic images and $D_{1} \in \mathscr{F}$, then $\mathscr{G}(R) \subseteq A_{1}$.

We proceed by induction to define $R e_{2}, R e_{3}, \cdots, R e_{n}$ as follows: Suppose $R e_{1}, \cdots, R e_{u}$ has been constructed such that

$$
R=R e_{1} \oplus \cdots \oplus R e_{u} \oplus A_{u},
$$

$\mathscr{G}(R) \subseteq A_{u}$, each $R e_{i}$ is a uniform left ideal, and

$$
1=e_{1}+e_{2}+\cdots+e_{u}+a_{u}, \quad a_{u} \in A_{u} .
$$

If $A_{u}=\mathscr{G}(R)$, we are done. If $A_{u} \neq \mathscr{G}(R)$, then let $R x \neq 0$ be a torsionfree uniform left ideal contained in $A_{u}$. If $R e_{i} x=0$ for all $i=1,2, \cdots, u$, then $A_{u} x=R x \in \mathscr{F}$. So the exact sequence

$$
0 \rightarrow(0: x) \cap A_{u} \rightarrow A_{u} \rightarrow A_{u} x \rightarrow 0
$$

splits since $A_{u} x$ is projective. Hence $A_{u}=D_{u+1} \oplus A_{u+1}$ where $D_{u+1} \cong$ $A_{u} x$. Write $a_{u}=e_{u+1}+a_{u+1}$ with $e_{u+1} \in D_{u+1}, a_{u+1} \in A_{u+1}$. Then $R=$ $R e_{1} \oplus \cdots \oplus R e_{u} \oplus R e_{u+1} \oplus A_{u+1}$, and $\mathscr{C}(R) \subseteq A_{u+1}$.

By (1) and Theorem 2.1(2), it follows that the above process 
must stop after finitely many steps (i.e., eventually we cannot assume $R e_{i} x=0$ for all $i$ as above). Hence we may assume that we have constructed uniform torsionfree left ideals $R e_{1}, R e_{2}, \cdots, R e_{n}$ with the properties:

(i ) $\quad R=R e_{1} \oplus \cdots \oplus R e_{n} \oplus A$.

(ii) $1=e_{1}+e_{2}+\cdots+e_{n}+a, a \in A$.

(iii) If $R x \subseteq A$ is a nonzero torsionfree uniform left ideal, then $R e_{i} x \neq 0$ for some $i \in\{1,2, \cdots, n\}$.

(iv) $\mathscr{G}(R) \subseteq A$.

Suppose $0 \neq R x \in \mathscr{F}, R x \subseteq A$, and $R x$ uniform. It is easily seen that $e_{i} e_{j}=0$ for $i \neq j$ and $e_{i} a=0 \forall i=1,2, \cdots, n$. So for $y \in R x$, $R e_{i} a y=0$. Since $R a y \leqq R x$, it follows from (iii) above that $a y=0$. Let $q$ be the least integer such that $R e_{q} x \neq 0$, which exists by (iii). It is easily verified that if $v \in \oplus \sum_{i \neq q} R e_{i} \oplus A$, then $v e_{q} x=0$. It follows that if $\left(0: e_{q} x\right) \cap R e_{q} \neq 0$, then $\left(0: e_{q} x\right)$ is essential in $R$, and hence $\left(0: e_{q} x\right) \in F(\mathscr{G})$. But then $R e_{q} x \in \mathscr{G} \cap \mathscr{F}=0$, a contradiction. Therefore, $\left(0: e_{q} x\right) \cap R e_{q}=0$.

Note that the conditions

(a) $\quad\left(0: e_{q} x\right) \supseteqq\left(\oplus \sum_{i \neq q} R e_{i}\right) \oplus A$

(b) $\left(0: e_{q} x\right) \cap R e_{q}=0$

(c) $\quad R=R e_{1} \oplus \cdots \oplus R e_{n} \oplus A$

imply that $\left(0: e_{q} x\right)=\left(\oplus \sum_{i \neq q} R e_{i}\right) \oplus A$, and therefore $R e_{q} x \cong R e_{q}$. Set $N_{1}=R e_{q} x$. Then $N_{1}^{2} \cong(R x a) \cdot R x=R x \cdot(a R x)=R x \cdot 0=0$.

If $A$ is not an essential extension of $N_{1} \oplus \mathscr{G}(R)$, then repeat the process used to obtain $N_{1}$ to get $N_{2} \subseteq A$ such that $N_{2}^{2}=0$ and $R e_{p} \cong N_{2}$ for some $p \in\{1,2, \cdots, n\}$. By induction we construct $N_{3}, N_{4}, \cdots$ with the desired properties. Moreover, by (1) and Theorem 2.1 this induction process stops after finitely many steps, say $m$. Thus $A$ is an essential extension of $N_{1} \oplus N_{2} \oplus \cdots \oplus N_{m} \oplus \mathscr{G}(R)$ as desired.

$(2) \Rightarrow(1)$ : This is an immediate consequence of Theorem 2.1 $(3) \Rightarrow(1)$.

The next theorem is the main result of this section.

THEOREM 3.3 The following are equivalent:

(1) $R=\mathscr{G}(R) \oplus R_{1} \oplus R_{2} \oplus \cdots \oplus R_{m}$, where $R_{i} R_{j}=0$ for $i \neq j$,

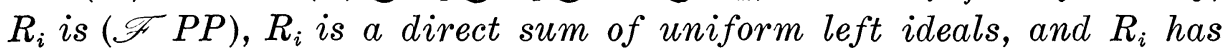
a simple classical left quotient ring with minimum conditions for $i=1,2, \cdots, m$.

(2) (i ) $R$ is (F्FPP).

(ii) $F(\mathscr{G})$ has a cofinal subset of finitely generated left ideals.

(iii) There are no nonzero torsionfree nilpotent left ideals in $R$.

$(1) \Rightarrow(2): \quad$ Write $R_{i}=R f_{i}$, where $1=f_{1}+f_{2}+\cdots+f_{m}+g$ 
and $g \in \mathscr{G}(R)$. We claim that any principal left ideal in $\mathscr{F}$ is $R$ isomorphic to a principal left ideal in $R f_{1}+R f_{2}+\cdots+R f_{m}$. For if $x=r_{1} f_{1}+\cdots+r_{m} f_{m}+r g$ generates a principal left ideal in $\mathscr{F}$, then the mapping of $R x$ induced by

$$
x \rightarrow r_{1} f_{1}+\cdots+r_{m} f_{m}
$$

has kernel $K=\{\operatorname{trg} \mid t \in R, \operatorname{trg} \in R x\}$. But then $R \operatorname{trg} \in \mathscr{G} \cap \mathscr{F}=0$. Thus $K=0$ and the claim is established.

Thus to show (i), it is sufficient to show that each principal left ideal in $R f_{1}+\cdots+R f_{m}$ is $R$-projective. Since each $R f_{i}=R_{i}$ is $(\mathscr{F} P P)$ and $f_{i} c f_{j}=0$ for $i \neq j(c \in R)$, this is easily verified using the fact that each $R_{i}$ is (FFPP).

Condition (ii) is immediate from Theorem 2.1 (3).

Looking at the projections of any torsionfree nilpotent left ideal into the $R_{i}^{\prime}$ s, we see that the images of these projections all must be nilpotent left ideals. Since each $R_{i}$ has a simple classical left quotient ring with minimum conditions, then it follows from [10], Theorem (p. 268), that each image is zero. It follows that zero is the only nilpotent torsionfree left ideal.

(2) $\Rightarrow(1)$ : By Theorem 3.2, we have

$$
R=R e_{1} \oplus R e_{2} \oplus \cdots \oplus R e_{n} \oplus A,
$$

where $A$ is an essential extension of $\mathscr{G}(R) \oplus N_{1} \oplus N_{2} \oplus \cdots N_{m}$ with $N_{i}^{2}=0$ for $i=1,2, \cdots, m$. From (iii) it follows that $A=\mathscr{G}(R)$. Hence $R=R e_{1} \oplus \cdots \oplus R e_{n} \oplus \mathscr{G}(R)$.

Define $i \sim j$ if either $e_{i} R e_{j} \neq 0$ or $e_{j} R e_{i} \neq 0$ for $1 \leqq i, j \leqq n$. It is easily verified that $\sim$ is an equivalence relation. Let $S(1), S(2), \cdots$, $S(m)$ be the distinct equivalence classes of $\{1,2, \cdots, n\}$ under $\sim$, and let $R_{i}=\sum_{j \in S(i)} R e_{j}$. Using Goldie's Theorem ([10], p. 268), the reader can verify that the $R_{i}$ have the required properties.

Note that " $(\mathscr{F} P P)$ " can be replaced by "finitely generated torsionfree left ideals are projective" or "torsionfree left ideals are projective" in the statement of Theorem 3.3, and the result remains true with only trivial modifications in the proof. In case $R$ has a semisimple left classical quotient ring with minimum conditions, then $\mathscr{G}(R)=0$, $R$ has no infinite direct sums of left ideals, and $R$ has no nonzero nilpotent left ideals. Hence the following result of L. Levy ([11], Th. 4.3) is a special case of Theorem 3.3.

COROLLARY 3.4. Let $R$ be a hereditary ring with semisimple left classical quotient ring $S$. Then $R$ is a direct sum of hereditary rings $\left\{R_{i} \mid i=1,2, \cdots, n\right\}$ which have simple left classical quotient rings with minimum condition. When considered as a set of left 
ideals of $R,\left\{R_{i} \mid i=1,2, \cdots, n\right\}$ constitutes a minimal set of annihilator ideals of $R$, and hence the quotient rings of the $R_{i}^{\prime}$ s are the simple components of $S$.

Since the concept of torsion defined by L. Levy [11] coincides with the Goldie torsion concept for rings possessing a semisimple left classical quotient ring with minimum conditions, the next corollary is a generalization of [11], Theorem 6.1.

COROLLARY 3.5. Suppose that the conditions of Theorem 3.3 hold with "(F्F PP)" replaced by "finitely generated left ideals in $\mathscr{F}$ are projective." Suppose $R / \mathscr{G}(R)$ has the ascending chain condition on annihilator right ideals, and suppose that $R$ contains no infinite direct sum of right ideals. Then every finitely generated $R$-module $M$ is a direct sum of $\mathscr{G}(M)$ and finitely many left ideals of $R$.

Proof. Under these hypotheses $R / \mathscr{G}(R)$ has a two-sided semisimple classical quotient ring with minimum conditions. Note that $R / \mathscr{G}(R)$ also possesses a Goldie torsion theory, which coincides with Levy's torsion theory for $R / \mathscr{G}(R)$. Let $M$ be a finitely generated $R$-module. Since $M / \mathscr{G}(M)$ is a $R / \mathscr{G}(R)$-module, it follows from [11], Theorem 5.2, that $M / \mathscr{G}(M)$ is isomorphic to a submodule of a free $R / \mathscr{G}(R)$-module. But by Theorem $3.3, R / \mathscr{G}(R)=R_{1}+R_{2}+\cdots+R_{m}$ (ring direct sum), where each $R_{i}$ is semi-hereditary. So [3] Theorem I.6.1 yields $M / \mathscr{G}(M) \cong \bigoplus \sum_{i=1}^{n} I_{i}$, where $I_{i}$ is a finitely generated left ideal of $R / \mathscr{G}(R)$. By Theorem 3.3, each $x \in R$ can be written uniquely as $r_{1}+r_{2}+\cdots+r_{m}+g$, where $r_{i} \in R_{i}$ and $g \in \mathscr{G}(R)$. Hence each $x^{\prime}=x+\mathscr{G}(R) \in R / \mathscr{G}(R)$ can be written uniquely as

$$
r_{1}+r_{2}+\cdots+r_{m}+\mathscr{G}(R) .
$$

Thus each $I_{i}$ is $R$-isomorphic to a finitely generated left ideal of $R$ contained $\sum_{i=1}^{m} R_{i}$ via

$$
r_{1}+r_{2}+\cdots+r_{m}+\mathscr{G}(R) \longrightarrow r_{1}+r_{2}+\cdots+r_{m} .
$$

So by Theorem 3.3, each $I_{i}$ is $R$-projective. Therefore,

$$
M \cong \mathscr{G}(M) \oplus I_{1} \oplus I_{2} \oplus \cdots \oplus I_{n} .
$$

An element $n$ of a left ideal $N$ of $R$ is said to be in the center modulo $\mathscr{G}(R)$ if the image of $n$ under the natural homomorphism $\rho: R \rightarrow R / \mathscr{G}(R)$ is a nonzero element of the center of the ring $R / \mathscr{G}(R)$. Equivalently, an element $n$ is in the center modulo $\mathscr{G}(R)$ if, for each $x \in R$, there exists an element $t_{x} \in \mathscr{G}(R)$ such that $x n=n x+t_{x}$. 


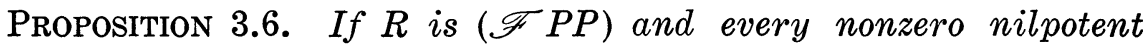
left ideal in $\mathscr{F}$ contains an element in the center modulo $\mathscr{G}(R)$, then in fact $R$ has no nilpotent left ideals in $\mathscr{F}$.

Proof. Assume otherwise. Then there exists $n \in R$ with the following properties:

(1) $R n \in \mathscr{F}$.

(2) $(R n)^{2}=0$.

(3) For each $x \in R$, there exists $t_{x} \in \mathscr{G}(R)$ such that $n x=x+t_{x}$. By $(\mathscr{F} P P)$, there is an isomorphism $\lambda: R n \rightarrow R e$, where $\mathrm{e}^{2}=e \neq 0$. Let $r \in R$ be such that $\lambda(r n)=e$. Then $n \in(0: r n)=(0: e)$, so that ern $=n e r+t=t$ for some $t \in \mathscr{G}(R)$. So (1) implies that $e \in(0: r n)=$ $(0: e)$, a contradiction.

CoROLlaRy 3.7. Let $R$ be commutative. Then the following are equivalent:

(1) $R$ is $(\mathscr{F} P P)$ and $F(\mathscr{G})$ has a cofinal subset of finitely generated left ideals.

(2) $R=\mathscr{G}(R)+R_{1}+R_{2}+\cdots+R_{n}$ (ring direct sum), where each $R_{i}$ is an integral domain.

Proof. Apply Proposition 3.6 and Theorem 3.3. Then note that a commutative prime ring is an integral domain. Conversely, $(2) \Rightarrow(1)$ follows from Theorem 3.3.

Recall that $R$ is $(P P)$ if and only if $R$ is $(\mathscr{F} P P)$ and $\mathscr{G}(R)=0$. Hence the following result of A. Hattori ([8], Lemma 3 ) is a special case of Corollary 3.7.

COROLlaRY 3.8. Let $R$ be a commutative ring having no infinite direct sum of left ideals. Then $R$ is a (PP) ring if and only if $R$ is a direct sum of integral domains.

The results in $\S 2$ and $\S 3$ of this paper will appear in the author's dissertation at the University of Nebraska. The author is deeply indebted to his adviser S. E. Dickson for his advice and encouragement. He is also grateful to J. S. Alin and E. P. Armendariz for several stimulating conversations.

\section{REFERENCES}

1. J. S. Alin, Thesis, University of Nebraska, 1967.

2. J. S. Alin and S. E. Dickson, Goldie's torsion theory and its derived functor, Pacific

J. Math. 24 (1968), p. 195-203,

3. H. Cartan and S. Eilenberg, Homological algebra, Princeton, 1955. 
4. C. Curtis and I. Reiner, Representation theory of finite groups and associate algebras, Wiley, 1962.

5. S. E. Dickson, A torsion theory for Abelian categories, Trans. Amer. Math. Soc. 121 (1966), 223-235.

6. A. W. Goldie, Semiprime rings with maximum condition, J. London Math. Soc.

(3) 10 (1960), 201-220.

7. — Torsion-free modules and rings, J. of Algebra 1 (1964), 268-287.

8. A. Hattori, $A$ foundation of Torsion theory for modules over general rings, Nagoya Math. J. (1960), 147-170.

9. J. P. Jans, Some aspects of torsion, Pacific J. Math. 15 (1965), 1249-1259.

10. N. Jacobson, Structure of rings, Amer. Math. Soc. Colloquium Publication \#37, Second Edition, Providence, 1964.

11. L. Levy, Torsion-free and divisible modules over non-integral domains, Canad. J. Math. 15 (1963), 132-151.

12. F. L. Sandomierski, Semisimple maximal quotient rings, Trans. Amer. Math. Soc. 128 (1967), 112-120.

13. M. Teply, Torsionfree injective modules Pacific J. Math. 28 (1969), 441-453.

14. C. Walker and E. A. Walker, Quotient categories and rings of quotients (to appear).

Received February 16, 1968.

UNIVERSITY OF FLORIDA

GainesVille Florida 



\section{PACIFIC JOURNAL OF MATHEMATICS}

\section{EDITORS}

H. ROYDEN
Stanford University
Stanford, California

\section{R. R. PHELPS}

University of Washington

Seattle, Washington 98105
J. DUGUNDJI

Department of Mathematics

University of Southern California

Los Angeles, California 90007

\section{RICHARD ARENS}

University of California

Los Angeles, California 90024

\section{ASSOCIATE EDITORS}

E. F. BeCKenbaCh
B. H. NEUMANN

F. WOLF
K. YoshidA

\section{SUPPORTING INSTITUTIONS}

\author{
UNIVERSITY OF BRITISH COLUMBIA \\ CALIFORNIA INSTITUTE OF TECHNOLOGY \\ UNIVERSITY OF CALIFORNIA \\ MONTANA STATE UNIVERSITY \\ UNIVERSITY OF NEVADA \\ NEW MEXICO STATE UNIVERSITY \\ OREGON STATE UNIVERSITY \\ UNIVERSITY OF OREGON \\ OSAKA UNIVERSITY \\ UNIVERSITY OF SOUTHERN CALIFORNIA
}

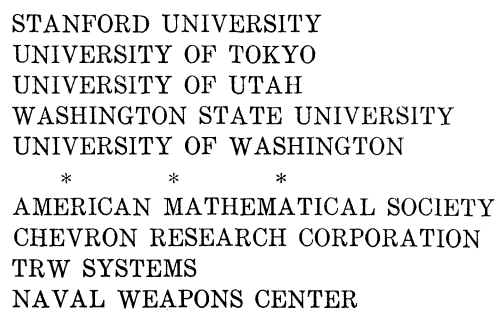

The Supporting Institutions listed above contribute to the cost of publication of this Journal, but they are not owners or publishers and have no responsibility for its content or policies.

Mathematical papers intended for publication in the Pacific Journal of Mathematics should be in typed form or offset-reproduced, double spaced with large margins. Underline Greek letters in red, German in green, and script in blue. The first paragraph or two must be capable of being used separately as a synopsis of the entire paper. It should not contain references to the bibliography. Manuscripts, in duplicate if possible, may be sent to any one of the four editors. Please classify according to the scheme of Math. Rev. 36, 1539-1546. All other communications to the editors should be addressed to the managing editor, Richard Arens, University of California, Los Angeles, California, 90024.

50 reprints are provided free for each article; additional copies may be obtained at cost in multiples of 50 .

The Pacific Journal of Mathematics is published monthly. Effective with Volume 16 the price per volume (3 numbers) is $\$ 8.00$; single issues, $\$ 3.00$. Special price for current issues to individual faculty members of supporting institutions and to individual members of the American Mathematical Society: $\$ 4.00$ per volume; single issues $\$ 1.50$. Back numbers are available.

Subscriptions, orders for back numbers, and changes of address should be sent to Pacific Journal of Mathematics, 103 Highland Boulevard, Berkeley, California, 94708.

PUBLISHED BY PACIFIC JOURNAL OF MATHEMATICS, A NON-PROFIT CORPORATION

Printed at Kokusai Bunken Insatsusha (International Academic Printing Co., Ltd.), 7-17, Fujimi 2-chome, Chiyoda-ku, Tokyo, Japan. 


\section{Pacific Journal of Mathematics \\ Vol. 29, No. $2 \quad$ June, 1969}

Bruce Langworthy Chalmers, On boundary behavior of the Bergman kernel function and related domain functionals ................... 243

William Eugene Coppage, Peirce decomposition in simple Lie-admissible power-associative rings .............................. 251

Edwin Duda, Compactness of mappings...................... 259

Earl F. Ecklund Jr., On prime divisors of the binomial coefficient......... 267

Don E. Edmondson, A modular topological lattice ............... 271

Phillip Alan Griffith, A note on a theorem of Hill ................... 279

Marcel Herzog, On finite groups with independent cyclic Sylow

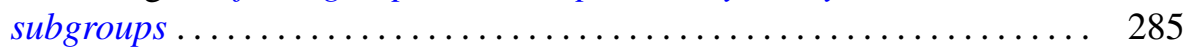

James A. Huckaba, Extensions of pseudo-valuations................. 295

S. A. Huq, Semivarieties and subfunctors of the identity functor ........ 303

I. Martin (Irving) Isaacs and Donald Steven Passman, Finite groups with small character degrees and large prime divisors. II ............ 311

Carl Kallina, A Green's function approach to perturbations of periodic

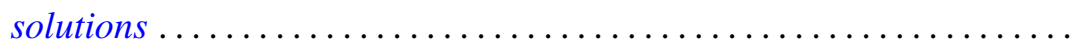

$\mathrm{Al}$ (Allen Frederick) Kelley, Jr., Analytic two-dimensional subcenter manifolds for systems with an integral ....................

Alistair H. Lachlan, Initial segments of one-one degrees ............ 351

Marion-Josephine Lim, Rank k Grassmann products ............. 367

Raymond J. McGivney and William Henry Ruckle, Multiplier algebras of

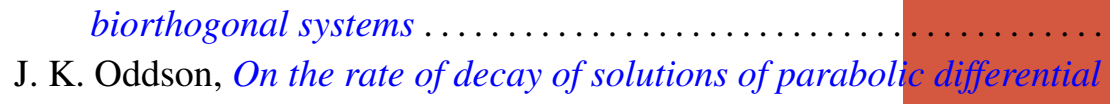

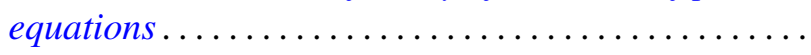

Helmut R. Salzmann, Geometries on surfaces ........... .

Annemarie Schlette, Artinian, almost abelian groups and their groups of automorphisms ............................

Edgar Lee Stout, Additional results on modules over polydisc algebras ...

Lajos Tamássy, A characteristic property of the sphere . .

Mark Lawrence Teply, Some aspects of Goldie's torsion theory. ...

Freddie Eugene Tidmore, Extremal structure of star-shaped sets ...

461

Leon Jarome Weill, Unconditional and shrinking bases in locally convex spaces... 